\title{
Urbain Jean Joseph Le Verrier
}

\begin{abstract}
Astronome et mathématicien français, Urbain Le Verrier est surtout connu pour ses travaux en mécanique céleste, qui lui ont permis de prédire l'existence de la planète Neptune par la seule puissance du calcul. II a également dirigé et profondément réformé l'Observatoire de Paris à la fin du $19^{\mathrm{e}}$ siècle, et fondé les services français de météorologie.
\end{abstract}

Riad HAIDAR, haidar@onera.fr

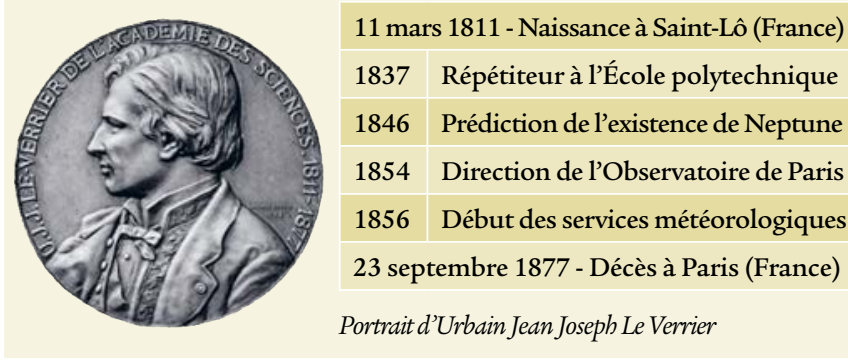

enfants. Lucile est une musicienne de talent, mais elle pratique en amateur. Les deux garçons du couple réussiront à leur tour le concours d'entrée à l'École polytechnique, et intégreront le prestigieux corps des Mines.

\section{Enseignement à l'École polytechnique}

L'objectif de Le Verrier est d'intégrer le corps enseignant de l'École polytechnique. Aussi, lorsque deux postes de répétiteur sont ouverts en 1837 , il candidate sans hésiter : sa préférence va au poste de chimie, aux côtés de Gay-Lussac, mais il se retrouve en concurrence avec Henri Victor Regnault, qui est le favori ; en revanche, Le Verrier décroche sans difficulté l'autre poste, en géodésie, avec Félix Savary. C'est un changement radical de domaine scientifique, puisqu'il passe de la chimie à l'astronomie et à la mécanique céleste, mais ses compétences en mathématiques suffisent à lever tous les doutes. Il est également nommé examinateur au concours d'entrée à l'École polytechnique. En 1840, l'état de santé de Savary se dégrade fortement; il décède en juillet 1841 et est alors remplacé par Michel Chasles, dont Le Verrier devient naturellement le répétiteur.

À son nouveau poste, et tout en enseignant, Le Verrier fait ses premières armes en travaillant sur le problème de la stabilité du système solaire. C'est avant tout un problème de maths - donc parfaitement à sa portée. Il publie ses premiers résultats Sur les variations séculaires des orbites des planètes dès 1839, dans les Comptes-Rendus de l'Académie des Sciences. Il montre également que le mouvement périodique des comètes obéit, comme celui des planètes, aux lois de l'attraction établies par Newton; et démontre la perturbation qu'exerce Jupiter sur certaines de leurs trajectoires.

Ses travaux attirent l'attention de François Arago qui, en 1845 , l'incite à s'intéresser à la planète Uranus. En effet, la trajectoire de cette planète géante de glaces, découverte en 1781 par William Herschel, recèle une énigme: son orbite montre des déviations notables par 
rapport aux paramètres théoriques, établies entre autres par Pierre-Simon de Laplace en 1783. Notons d'ailleurs qu'en 1841 et de l'autre côté de la Manche, John Couch Adams avait émis l'hypothèse qu'une planète inconnue serait la cause des perturbations constatées... mais sans parvenir à le montrer. Voilà un défi à la hauteur du talent de Le Verrier, qui se prend au jeu. Il établira ses résultats en trois actes, avec un raffinement croissant. Acte I : en décembre 1845, il publie un premier mémoire dans lequel il détermine l'influence que Jupiter et Saturne exercent sur la trajectoire d'Uranus. Ces travaux, d'une haute technicité mathématique, sont proprement remarquables; et ils sont remarqués : ils lui valent d'ailleurs une renommée immédiate, puisqu'il est élu à l'Académie des Sciences, en section d'astronomie, le 19 janvier 1846 . Acte II : le $1^{\text {er }}$ juin 1846, il démontre que les perturbations d'Uranus ne peuvent être dues aux planètes déjà connues, et il en déduit l'existence d'une nouvelle planète, qui se situerait au-delà de l'orbite d'Uranus. Acte III : deux mois plus tard, il va encore plus loin et décrit en détail l'orbite de cette planète encore hypothétique.

\section{Découverte de Neptune}

Mais il reste à asséner la preuve ultime, et Le Verrier semble avoir une idée précise du meilleur scénario. Le 18 septembre 1846, il écrit à Johann Galle, jeune astronome à l'Observatoire de Berlin. Il lui demande, tout de go et en substance, de pointer son télescope vers l'endroit du ciel que ses calculs indiquent. Galle hésite : la requête est peu commune, voire absolument inédite ; l'effort à fournir n'est pas anodin, pour un succès très (peut-être trop) peu probable. Pourtant Le Verrier montre une telle confiance dans ses calculs que l'astronome berlinois accepte. Avec l'accord de ses responsables, le soir même qui suit la réception de la lettre du mathématicien français, il cherche la planète prédite et la trouve à moins d'un degré de la position calculée. C'est proprement spectaculaire! Pourtant, en scientifique aguerri, Galle ne s'emballe pas : il attend la nuit suivante pour, soigneusement, vérifier ses premières observations et confirmer qu'il s'agit bien d'une planète. Alors seulement, absolument sûr de ses résultats, il diffuse la nouvelle... qui fait un bruit de tonnerre !

Le Verrier est immédiatement projeté sur le devant de la scène. Le Bureau des longitudes le nomme membre adjoint ; il reçoit la Légion d'Honneur ; à Londres, la Royal Society lui décerne sa prestigieuse Copley Medal, et le London Times titre en une Le Verrier's planet found. D'ailleurs, il faut bien trouver un nom à cette nouvelle planète : ce sera Neptune.

En 1847, une chaire de mécanique céleste est créée pour lui à la Sorbonne. À ce poste, il s'embarque dans un projet ambitieux, qu'il poursuivra jusqu'à sa mort : l'élaboration d'une théorie complète du système solaire. Il montre notamment une faible anomalie de la trajectoire de Mercure, qui sera imputée à l'existence d'une nouvelle planète, plus proche du soleil que Mercure, et baptisée Vulcain. Cette hypothèse, après avoir été activement étudiée, sera finalement abandonnée au $20^{\mathrm{e}}$ siècle, et l'explication de l'anomalie de Mercure sera un des premiers succès de la théorie de la Relativité générale... Les travaux de Le Verrier sont proprement titanesques, et la Royal Astronomical Society ne s'y trompe pas : elle lui décerne, par deux fois, sa plus haute distinction, la Gold Medal, en 1868 puis en 1876.

\section{Observatoire de Paris, et Météorologie}

En 1854, Le Verrier succède à Arago à la tête de l'Observatoire de Paris, dont il fait un diagnostic sévère et pour lequel il nourrit de grandes ambitions... Sous sa direction, l'auguste institution doit se plier à une discipline et une organisation rigoureuses, inspirées du modèle de l'Observatoire britannique de Greenwich - mais Le Verrier est trop brutal dans sa volonté de réforme, et le personnel se rebelle. En 1870, à la suite de la démission collective de 14 astronomes, il est relevé de son poste et remplacé par Charles Delaunay. Mais Delaunay décède en 1872, et Le Verrier revient aux fonctions, non sans avoir dû accepter une concession majeure : son autorité sera désormais bridée par un conseil de laboratoire...

Malgré ces tensions, Le Verrier aura imprimé sa marque à la tête de l'Observatoire : c'est lui qui promeut l'utilisation du télégraphe, notamment pour la distribution de l'heure standard, et plus tard des avertissements météorologiques. Dès 1856, en effet, certains postes télégraphiques transmettent en temps réel leur observations météorologiques à l'Observatoire de Paris, qui publie un Bulletin quotidien repris par les journaux. Cette initiative donne naissance en 1878 au Bureau central météorologique, qui deviendra plus tard notre Météo France.

\section{Ultima verbae}

Le Verrier commence à souffrir du foie en 1873. Sa santé se détériore progressivement, et il décède le 23 septembre 1877 à l'Observatoire de Paris. Il est inhumé au cimetière du Montparnasse, sous une pierre surmontée d'un majestueux globe céleste en pierre. Mathématicien prodigieux, Le Verrier aura profondément réformé l'Observatoire de Paris et fondé la météorologie française, mais sa personnalité autoritaire continue, encore aujourd'hui, à susciter une appréciation contrastée de sa légende.

\section{POUR EN SAVOIR PLUS}

[1]James Lequeux, Le Verrier: savant magnifique et détesté(EDP sciences, 2009)

[2] Fabien Locher, Biographie d'U. Le Verrier, https://francearchives.fr/ commemo/recueil-2011/39833

[3] J.J. O'Connor, E.F. Robertson, MacTutor History of Mathematics (2014) 